\title{
Alcoholism and Catatonia: An Underappreciated Relationship
}

\section{Caitlin Snow, Daniela Guisado and Dimitry Francois}

Department of Psychiatry, Weill Cornell Medicine, USA

*Corresponding author: Dimitry Francois, Department of Psychiatry, Weill Cornell Medicine, New York Presbyterian/Weschester Division, 21 Bloomingdale Road, White Plains, NY 10605, USA, Tel: 914997 4375; Fax: 914682 6963; E-mail: dif9013@med.cornell.edu

Rec date: May 17, 2016; Acc date: Jun 06, 2016; Pub date: Jun 09, 2016

Copyright: () 2016 Snow C, et al. This is an open-access article distributed under the terms of the Creative Commons Attribution License, which permits unrestricted use, distribution, and reproduction in any medium, provided the original author and source are credited.

\section{Introduction}

Catatonia is a complex neuropsychiatric syndrome that has been described in medical literature since the 16th century [1]. Lifethreatening complications involving every organ system can ensue, signaling high levels of associated morbidity and mortality [2]. To this day, little is known about the etiology of catatonia [3]. Catatonia occurs most frequently in patients with affective disorders and schizophrenia, and is more common in medical or neurologic conditions [1]. However, there is a dearth of literature describing the relationship between alcohol use disorders and catatonia. Here we present the case of an older woman admitted for catatonia in the context of a history of alcohol use and Bipolar I disorder. We propose that her alcohol use could be a factor contributing to the onset of catatonia.

\section{Case Report}

The patient, a right handed 74-year-old woman with a long history of Bipolar I Disorder and Alcohol Use Disorder, was admitted for a three month psychiatric decline characterized by worsening depression, paranoia and catatonia. Collateral information revealed that the patient had been increasingly depressed with diminished energy, poor sleep, loss of appetite resulting in 15 pound weight loss, and paranoid thoughts that her daughters were poisoning her food. In the two weeks prior to admission she developed episodic catatonic symptoms of mutism, catalepsy, staring and withdrawal. She had a long history of consuming 7-10 standard alcoholic drinks daily until abruptly stopping one month prior to the onset of illness.

The patient's community psychiatrist treated her with various antidepressants (sertraline, venlafaxine, mirtazapine, and bupropion), mood stabilizers (lamotrigine, valproic acid, lithium) and antipsychotics (risperidone, olanzapine, aripiprazole) without improvement and with subsequent aggravation of her condition. Initial laboratory assessment (including complete blood count, electrolytes, renal and liver function tests) was within normal limits. Results on urine drug screen test were negative, and blood ethanol levels were undetectable. Electrocardiogram results were normal, and a brain computerized tomography scan did not demonstrate any pathology. She scored a 10 on the Bush-Francis Catatonia Rating Scale 4 and 27 on the Hamilton Depression Rating Scale $[4,5]$.

In view of the patient's lack of response to multiple psychotropic trials, electroconvulsive therapy (ECT) was recommended but the patient's family opted for ongoing medication management. The patient was treated with lorazepam up to $6 \mathrm{mg}$ daily, nortriptyline 70 $\mathrm{mg}$ daily with level of $110 \mathrm{ng} / \mathrm{mL}$ and lurasidone $60 \mathrm{mg}$ daily. After three weeks the patient's catatonia resolved (Bush-Francis Catatonia Rating Scale score of 0) and her depressive symptoms improved considerably (Hamilton Depression Rating Scale score of 7). The authors do not know if the patient sustained improvement following discharge.

\section{Discussion}

Although the exact mechanisms remain undefined, substantial evidence has implicated the dysregulation of the inhibitory $\gamma$ aminobutyric acid A (GABA-A), excitatory glutamate, and dopamine neurotransmitter systems as fundamental to the development of both catatonia and the sequelae of alcoholism [6-10]. In our case we speculate that chronic alcoholism lead to chronic brain changes and the abrupt stop of alcohol use triggered catatonia. It's important to note that our patient had a long history of bipolar disorder but never developed catatonia until she quit drinking.

Several case reports have identified co-occurring alcohol withdrawal and catatonia in humans. The first reported case of catatonia driven by alcohol withdrawal was described in 2007 [6]. Subsequent reports have described a woman with Bipolar II Disorder who presented with recurrent catatonia following alcohol withdrawal [7], a man with catatonia as the presenting feature of alcohol withdrawal [8], a woman with catatonia as a complication of alcohol withdrawal during the benzodiazepine taper period [9], and a man with catatonia in the setting of mixed alcohol and benzodiazepine withdrawal [10], Some authors have referenced the intriguing observation that dysregulation of the same neurotransmitter circuits have been implicated in the pathophysiology of both syndromes, but none have elaborated on this theory.

Alcohol exposure impacts the majority of neurochemical systems in the brain $[10,11]$. Although the precise neurobiological changes are complex and incompletely understood, imbalance of the GABAergic, glutaminergic, and dopaminergic systems are thought to play a central role $[9,10]$. Chronic alcohol exposure promotes compensatory neuroadaptations to oppose these effects and result in system of GABA-A hypoactivity, glutamate hyperactivity, and dopamine hypoactivity $[9,10]$. It is these neurochemical changes that may promote catatonia [11].

\section{Conclusion}

This article provides a neurobiological framework to support the hypothesis that alcoholism is an under recognized risk factor for developing catatonia, a thesis which may carry prognostic, treatment and research implications.

\section{References}

1. Fink M, Taylor MA (2006) Catatonia: a clinician's guide to diagnosis and treatment. Cambridge University Press, UK. 
Citation: Snow C, Guisado D, Francois D (2016) Alcoholism and Catatonia: An Underappreciated Relationship. J Gerontol Geriatr Res 5: 314. doi:10.4172/2167-7182.1000314

Page 2 of 2

2. Gross AF, Smith FA, and Stern TA (2008) Dread Complications of catatonia: A case discussion and review of the literature. Prim Care Companion. J Clin Psychiatry 10: 153-155.

3. Daniels J (2009) Catatonia: Clinical aspects and neurobiological correlates. J Neuropsychiatry Clin Neurosci 21: 371-380.

4. Bush G, Fink M, Petrides G, Dowling F, Francis A (1996) Catatonia. I. Rating scale and standardized examination. Acta Psychiatr Scand 93: 129-136.

5. Hamilton MAX (1967) Development of a rating scale for primary depressive illness. Br J Soc Clin Psychol 6: 278-296.

6. Muralidharan K, Rajkumar RP, Ananthapadmanabha RS, Benegal V (2007) Catatonia as a presenting Feature of alcohol withdrawal: A Case report. Prim Care Companion J Clin Psychiatry 9: 465
7. Cottencin O, Danel T, Goudemand M, Thomas P, Consoli SM (2009) Catatonia recognition and treatment. Med Sci Monit 15:129-1231.

8. Narayanaswamy JC, Viswanath B, Jose SP, Benegal V (2011) Catatonia in alcohol withdrawal: a case report. Psychopathology 44: 136.

9. Geoffroy PA, Rolland B, Cottencin O (2012) Catatonia and Alcohol withdrawal: A complex and underestimated syndrome. Alcohol and Alcoholism 47: 288-290.

10. Basu A, Jagtiani A, Gupta R (2014) Catatonia in mixed alcohol and benzodiazepine withdrawal. J Pharmacol Pharmacother 5: 261-264.

11. Northoff G (2002) What catatonia can tell us about top-down modulation: a neuropsychiatric hypothesis. Behav Brain Sci 25: 555-604 